Historic, archived document

Do not assume content reflects current scientific knowledge, policies, or practices. 



\section{MIXSON'S FIELD SEEDS}

All selected, recieaned and packed in burlap bags without charge. Some Clovers and Grasses are packed in Cotton seamless bags, charged extra.

\section{SEED OATS}

\begin{tabular}{|c|c|c|}
\hline \multirow{2}{*}{\multicolumn{3}{|c|}{ 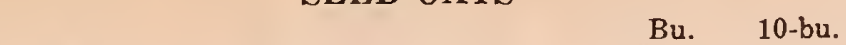 }} \\
\hline & & 10-bu. \\
\hline $\begin{array}{l}\text { Fulghum } \\
\text { Texas Red Rust } \text { (S. C. Grown }) \ldots \ldots \ldots \ldots\end{array}$ & $\$ 1.15$ & $\$ 1.10$ \\
\hline Burt or $90-\mathrm{Day} . . .$. & & .95 \\
\hline RYE & & \\
\hline Abbruzzi & 2.15 & 2.10 \\
\hline SEED CORN & & \\
\hline Mixson's Eureka (Special) & 2.75 & 2.65 \\
\hline Improved White Dent & 2.10 & 2.0 \\
\hline Early White Dent .... & 2.00 & 1.9 \\
\hline Improved Golden Dent & 2.25 & 2.1 \\
\hline Early Yellow Dent & 2.00 & 1. \\
\hline Tennessee Red Cob & 2.1 & 2.0 \\
\hline Paymaster & 2.1( & 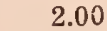 \\
\hline Mixson's Six Ear Prolific & 2.50 & 2. \\
\hline Batts Prolific & 2.25 & 2.1 \\
\hline Mosby's Prolific & 2.40 & 2.30 \\
\hline 保 & & \\
\hline
\end{tabular}

\section{COTTON SEED}

\begin{tabular}{|c|c|c|c|c|}
\hline \\
\hline & To & 10 & 26 to & 51 to \\
\hline & $10-\mathrm{bu}$ & 25-bu & $50 \mathrm{bu}$ & $100 \mathrm{bu}$ \\
\hline Simpkin's Ideal & 1.75 & 1.65 & 1.55 & 1.45 \\
\hline King Early & 1.75 & 1.65 & 1.55 & 1.45 \\
\hline Wannamaker-Cleveland & 1.80 & 1.70 & 1.60 & 1.50 \\
\hline Mixson's Ex. Ey. Big Boll & 1.80 & 1.70 & 1.60 & 1. \\
\hline Simpkin's Bigg Boll...... & 1.75 & 1.65 & 1.55 & 1. \\
\hline Poulnot $\ldots \ldots \ldots \ldots \ldots$ & 1.75 & 1.65 & 1.55 & 1.4 \\
\hline Pool's Big Boll........... & 1.75 & 1.65 & 1.55 & 1 \\
\hline Webber No. 4!) Strain $6 \ldots$ & 1.75 & 1.65 & 1.55 & 1 \\
\hline Deltatype Webber ...... & 1.80 & 1.70 & 1.60 & \\
\hline Lightning Express & 1.80 & 1.70 & 1.60 & \\
\hline Hartsville Iong Staple ... & 1.70 & 1.65 & 1.55 & \\
\hline nbia Staple ........ & 1.75 & 1.65 & 1.5 & \\
\hline Mixson's W' L. Staple.... & 1.80 & $\begin{array}{l}1.70 \\
-\mathrm{AS}\end{array}$ & 1.60 & \\
\hline 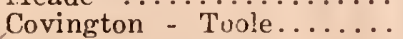 & 1.8 & 1.70 & & \\
\hline maker & 1.7 & 1.65 & 1.5 & \\
\hline Toole - Prolific. & 1.80 & 1.70 & 1.60 & \\
\hline Half and Half. & 1.80 & 1.70 & 1.60 & \\
\hline Bank Account & 1.75 & 1.65 & 1.55 & \\
\hline well & 1.75 & 1.65 & 1.55 & \\
\hline Mexican Big & 1.75 & 1.65 & 1.55 & \\
\hline
\end{tabular}

\section{CLOVERS}

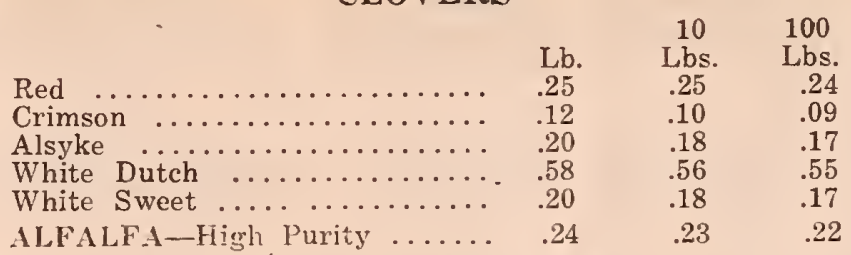

\section{PEANUTS}

All recleaned, stemn:ed, and hand picked.

10Lts. 100Lbs.

White Spanish $\ldots \ldots \ldots \ldots \ldots \ldots \ldots \ldots \ldots . .14 \quad .12^{1}{ }_{2}$

N. C Runner $\ldots \ldots \ldots .121 / 2$

Red Spanish ......................... $14 \quad .12^{1 / 2}$

Valentia ........................... $.15 \quad .13^{1 \%}$

\begin{tabular}{|c|c|c|c|}
\hline \multicolumn{4}{|c|}{ VETCH } \\
\hline & Lb. & $\begin{array}{c}10 \\
\text { Lbs. }\end{array}$ & $\begin{array}{l}100 \\
\text { Lbs. }\end{array}$ \\
\hline Hairy & .16 & .14 & \\
\hline $\begin{array}{l}\text { Oregon } \\
\text { Augusta }\end{array}$ & .10 & $\begin{array}{l}.08 \\
.07\end{array}$ & . \\
\hline
\end{tabular}

\section{SORGHUM OR CANE SEED}

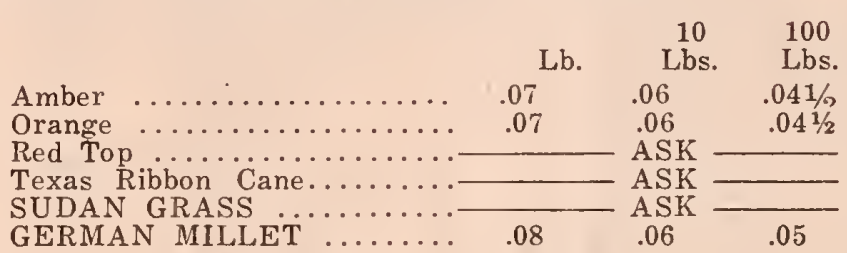

\section{MISCELLANEOUS FEED SEEDS}

Italian Rye Grass ................ $17 \quad .15$

Tall Meadow Oat Grass ....... $\quad .25 \quad .23 \quad .22$

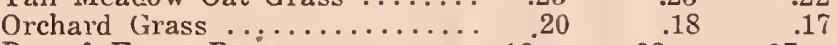

Dwarf Essex Rape .............. ${ }^{.0} \quad .08 \quad .07$

Bermuda Grass ........... $\quad .37 \quad 35 \quad 34$

Pearl or Cat Tail Millet..... $\quad .14 \quad .12 \quad .111 \%$

Mam. Russian Sunflower $\ldots \ldots . .12 \quad .10 \quad .09$

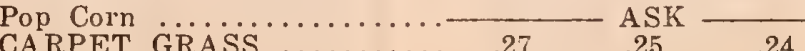

DALLIS GRASS $\ldots \ldots \ldots \ldots \ldots, \quad .83 \quad .81 \quad .80$

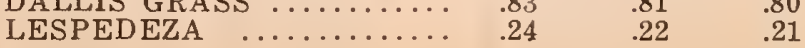



